\title{
Shoji Itakura, Kazuo Fujita (eds.): Origins of the social mind: evolutionary and developmental views
}

\author{
Springer, Tokyo, 2008, 211pp
}

\author{
Henrike Moll
}

Received: 25 March 2009/Accepted: 28 March 2009/Published online: 23 April 2009

(C) Japan Monkey Centre and Springer 2009

Origins of the Social Mind is a collection of papers that unites the latest empirical findings and conceptual insights from cognitive development, and primate and avian cognition, written by some of the leading researchers in the respective fields. As the editors mention in the preface, there has recently been a trend for transdisciplinary cooperation among primatologists and developmental psychologists - and this is one of the few anthologies that provides an excellent representation of this rapprochement.

One striking aspect is the great diversity that is covered in terms of the species investigated (ranging from corvids, parrots, monkeys, and apes to humans), age groups tested (from infants to adults), and research methods employed (from naturalistic observations to experiments and interviews). Importantly, this variability does not result in unrelated pieces that lack a common topical focus; quite the contrary, the reader quickly identifies the recurring themes in this book to be cooperation, competition, and communication in the animal kingdom and human life.

Origins of the Social Mind comprises nine chapters and a closing commentary by one of the editors, Shoji Itakura. The chapters are organized into two parts. The first part (Chaps. 1-5) deals with the phylogeny of cooperative activities, maternal behaviors, and various "mind-reading" abilities. The second part (Chaps. 6-9, plus commentary)

H. Moll

Max Planck Institute for Evolutionary Anthropology,

Leipzig, Germany

Present Address:

H. Moll $(\bowtie)$

Institute for Learning and Brain Sciences,

University of Washington, Seattle, USA

e-mail: moll@eva.mpg.de revolves around the development of various social-cognitive abilities in humans. Interestingly, the majority of these chapters deal with skills that are not among the usual suspects in the literature about theory of mind and related topics, namely: negotiating with others to achieve a consensus, impression management, and "acting as if" in the context of performance art.

\section{Part 1: Phylogeny of social cognition}

One noteworthy aspect is the emphasis that is placed on ecological validity throughout the first part of the book. The animals in the experiments were not confronted with tasks that seem to have been designed for human subjects. Instead, animals were tested in very natural scenarios, such as competing with others over valuable resources (monkeys, Chap. 1), caching food to protect it from potential pilferers (corvids, Chap. 3), and coordinating behaviors with others to make resources accessible that cannot be obtained by individual action alone (parrots, Chap. 5).

The journey begins with a rich amalgam of studies with capuchin monkeys, Cebus apella, conducted at the Primate Research Institute in Kyoto. Kazuo Fujita, Hika Kuroshima, Yuko Hattori, and Makoto Takahashi report some impressive results showing traces of deceptive behavior, cooperation, and knowledge attribution in these monkeys. The fact that New World monkeys display such sophisticated forms of social cognition may lead to a reconsideration of the phylogenetic onset of these skills. It may also generate a line of research that aims to identify and compare the specific ways in which the different species approach these tasks, as well as the kind and amount of training that is required for them to succeed in these tests. 
In Chap. 2, Satoshi Hirata gives a sensible and thoughtful description of early dyadic interactions between chimpanzee mothers and their infants_-both in the wild and in captivity. One may expect core maternal behaviors such as nursing and protecting the young to be in-built and stable across different environmental conditions. However, as Hirata demonstrates in his examples, chimpanzees who were raised by humans can be challenged by these basic maternal tasks to a degree that they seek help from humans. An important question that these observations raise is whether this seeking of assistance reflects a lack of critical learning experiences acquired in the wild, or if humanreared primates build special relations with the humans that allow them to accept these humans as caregivers for their child.

Moving from dyadic to triadic engagement in Chap. 3, James Anderson and Sarah-Jane Vick give an interesting review of gaze understanding in the primate species. They provide a fine-grained picture of the state of the art and point at the relevance of factors such as rank, socioecology, upbringing, and the specifics of the experimental setup. One interesting phenomenon is a dissociation found in apes, but not humans; whereas apes seem unable to use their gaze understanding in communicative scenarios, such as the object choice task, human infants quickly learn that another may use his gaze to give them a hint or a wink. The authors' analyses will hopefully foster many more research projects looking at this and other questions revolving around eye contact, gaze, and their meaning in different species and contexts.

Nathan Emery and Nicola Clayton from the Cambridge Avian Cognition group remind us in Chap. 4 of the shortcomings of any anthropo- or primatocentric bias in comparative psychology. Birds' cognitive capacities have long been underestimated, partly due to a misconception of the structure of their brains. The series of experiments reported by Emery and Clayton suggest that Western scrub-jays preferably cache food in places that are (1) shady (versus well lit), (2) distant from (versus close to) an onlooker and potential pilferer, and (3) not visible (versus visible) for another individual. Food items that have been cached in "risky places" are relocated to safer places at a later time. Irrespective of whether these data speak in favor of a theory of mind in these corvids, they certainly demonstrate a level of social-cognitive skills that many did not expect.

With a similar emphasis on the relevance of ecological validity, Ludwig Huber, Gyula Gajdon, Ira Federspiel, and Dagmar Werdenich in Chap. 5 report some clever experiments with kea. These parrots live in steep, alpine habitats with limited access to resources-with the effect that cooperative strategies may be highly beneficial. In a number of experiments with various problem-solving scenarios, the researchers found that dominant birds "coerced" subordinate individuals into manipulating apparatuses such that the dominants could obtain the food reward. Future research should be aimed at identifying role reversal and situations in which two or more individuals truly have to work together (instead of one individual having to do all the work, while the other awaits the reward) to find out if kea can go beyond what the authors oxymoronically call "coercive cooperation."

\section{Part 2: Ontogeny of social cognition}

This part begins with an interesting chapter by Hanae Ando and Masuo Koyasu about some surprising differences in the acting skills of novices and expert actors. Not so surprising is the finding that adults evaluated the performances by professionals as generally better in quality and more realistic than those by novices. However, there was one striking piece of finding: novices outperformed experts in scenes in which they had to act as a person who is pretending to be in pain rather than simply a person in pain. The audience was unable to tell the difference between these two cases when the actors were experts, but had no problem perceiving the difference when the actors were novices. It thus seems that having received extensive training in "acting as if" makes it hard for experts to convey one thing on stage: namely "acting as if"-possibly because they have become so convincingly good at it that it cannot be distinguished from reality.

In Chap. 7, Philippe Rochat and Cláudia Passos Ferreira identify as the differentia specifica the ability of humans to negotiate with others. According to this view, Homo sapiens is not characterized best as rational animal or zoon logon echon, but as Homo negotiatus, who seeks to arrive at agreements with others. Objects of negotiation are not just the values of things etc., but also one's self-image as seen through the looking glass (Cooley 1902). Therefore, the ability to bargain and negotiate is tied to a particular sense of self - a self that is aware of its being subjected to others' judgments and evaluations and that cares about its reputation. The roots of negotiation and self knowledge lie, so it is argued, in an early sense of ownership and possession emerging at around 2 years of age.

In Chap. 8, Victoria Talwar and Kang Lee take a closer look at what is mostly considered a misconduct, but needs to be acknowledged as a significant cognitive achievement: telling lies. Even prior to an understanding of false beliefs, toddlers between 2 and 3 years sometimes make untrue statements after having committed a transgression. Soon thereafter, children also tell "white" lies to conceal another person's wrongdoing. However, these initial lies are easily detected by adults as they are poorly crafted and not well 
integrated with other statements. As their theory of mind abilities advance, children also begin to lie in more mature and clever ways.

In the last chapter, Shoji Itakura, Mako Okanda, and Yusuke Moriguchi report a set of interesting studies with infants in diverse areas of social cognition. Studies include investigations of young infants' sensitivity to social contingency, social influences on preschoolers' inhibitory control, and the attribution of dispositional qualities to geometric figures. For example, a very valuable control condition was added to the well-known helper-hinderer paradigm developed by Kuhlmeier et al. (2003) which has suggested an ability of infants to attribute dispositional qualities to others. This new control condition should lead into a renewed discussion about whether such a high-level interpretation is warranted, as the infants behaved just as they did in the experimental condition.

Finally, in his closing commentary, Shoji Itakura proposes an integrative model of social-cognitive development.
Some of the milestones acquired along the way are newborns' preference for faces and a propensity to imitate shortly after birth, followed by joint attention at 9 months of age, false-belief understanding at 4 years, and an appreciation of higher-order sarcasm at around 9 years of age.

Overall, Shoji Itakura and Kazuo Fujita edited a valuable, interesting book. Its comprehensiveness and the novelty of the research ensure its appeal to anyone with a vested interest in the question of how the social mind develops.

\section{References}

Cooley CH (1902) Human nature and the social order. Scribner's, New York

Kuhlmeier VA, Wynn K, Bloom P (2003) Attribution of dispositional states by 12-month-olds. Psychol Sci 14(5):402-408 